See discussions, stats, and author profiles for this publication at: https://www.researchgate.net/publication/307606609

\title{
A Multi-Start Simheuristic for the Stochastic Two-Dimensional Vehicle Routing Problem
}

Conference Paper · December 2016

DOI: 10.1109/WSC.2016.7822273

CITATIONS

6

4 authors, including:
READS

130

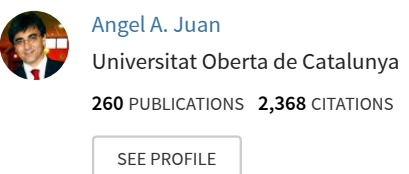

Some of the authors of this publication are also working on these related projects:

Project Master Thesis View project

Project Simulation and Analysis of Container Freight Train Operations at Port Botany View project 


\title{
A MULTI-START SIMHEURISTIC FOR THE STOCHASTIC TWO-DIMENSIONAL VEHICLE ROUTING PROBLEM
}

\author{
Daniel Guimarans \\ Aviation Academy \\ Amsterdam University of Applied Sciences \\ Weesperzijde 190 \\ Amsterdam, 1097 DZ, NETHERLANDS
}

\author{
Oscar Dominguez \\ Institute of Intelligent Systems and \\ Numerical Applications in Engineering \\ University of Las Palmas de Gran Canaria \\ Campus Universitario de Tafira \\ Las Palmas de Gran Canaria, 35017, SPAIN
}

\author{
Angel A. Juan \\ Enoc Martinez \\ Computer Science Dept. - IN3 \\ Open University of Catalonia \\ 5 Carl Friedrich Gauss Av. \\ Castelldefels, 08860, SPAIN
}

\begin{abstract}
The two-dimensional vehicle routing problem (2L-VRP) is a realistic extension of the classical vehicle routing problem where customers' demands are composed by sets of non-stackable items. Examples of such problems can be found in many real-life applications, e.g. furniture or industrial machinery transportation. Often, these real-life instances have to deal with uncertainty in many aspects of the problem, such as variable traveling times due to traffic conditions or customers availability. We present a hybrid simheuristic algorithm that combines biased-randomized routing and packing heuristics within a multi-start framework. Monte Carlo simulation is used to deal with uncertainty at different stages of the search process. With the goal of minimizing total expected cost, we use this methodology to solve a set of stochastic instances of the $2 \mathrm{~L}-\mathrm{VRP}$ with unrestricted oriented loading. Our results show that accounting for systems variability during the algorithm search yields more robust solutions with lower expected costs.
\end{abstract}

\section{INTRODUCTION}

The vehicle routing problem (VRP) is a well-known combinatorial optimization problem in which a fleet of vehicles has to service a set of customers at the lowest possible cost (Toth and Vigo 2014, Golden et al. 2008). The most basic variant of the VRP is the capacitated vehicle routing problem (CVRP), where an homogeneous fleet of vehicles with restricted capacity, based at a central depot, needs to satisfy customers' demands by visiting them only once. Additional restrictions, such as distance or time-based constraints, are often considered in richer variants of the problem. The CVRP and richer versions have been extensively studied due to their challenging NP-Hard nature and their potential applicability on real-life transportation activities (Caceres-Cruz et al. 2014, Lahyani et al. 2015).

In this work, we consider a realistic variant of the CVRP that combines vehicle routing and loading (packing) constraints, known as two-dimensional VRP (2L-VRP) (Iori et al. 2007). In this variant, customers' 
demands consists of a set of rectangular items that cannot be stacked, e.g., due to their weight or fragility. Our work was originally motivated by operations at Opein, a medium-sized company that hires industrial equipment to its customers, mostly in the construction field. Opein has to periodically deliver and pick up a variety of industrial machinery (e.g., aerial-work platforms, energy-generation sets, dumpers, forklifts, or professional cleaning equipment). Similar issues are also present in other transportation activities, where large-sized items are required to be picked up or delivered, e.g., furniture or appliances. These items must be efficiently accommodated in the truck to ensure high vehicle's utilization. This packing process may also critically affect routing decisions. Hence, one needs to consider not only the items weight, but also their dimensions. In the 2L-VRP, items are normally considered to be of rectangular shape and they cannot be piled up or overlap on the truck's loading surface.

One of the main reported issues in real operations is the significant variability on the time required to perform all the assigned activities. Although routes may be planned carefully, traveling time between customers depends on traffic and access conditions to the site, causing the actual time to vary substantially with respect to the original estimate. Likewise, customers' availability may also delay the start of the service. These deviations from the original plan translate into an increase of operational costs. Often, drivers need to be paid overtime due to an excess of driving hours beyond the duration of their shift. Furthermore, the company may incur into additional costs related to extending their operations time, e.g., paperwork needs to be finished after the completion of all routes.

In this paper, we present a hybrid simheuristic (Juan et al. 2015) for minimizing expected costs in the 2L-VRP with stochastic traveling times. We use a multi-start framework, where we embed biasedrandomized versions of classical routing and packing heuristics. Biased randomization of heuristics refers to the use of skewed probability distributions to induce an oriented (biased) random behavior of the heuristic, transforming a deterministic method into a probabilistic algorithm (Juan et al. 2013), (Juan et al. 2014). In our approach, we use a biased-randomized version of the classical Clarke and Wright savings heuristic for the CVRP (Clarke and Wright 1964), enhanced with memory-based techniques (Juan et al. 2010). To compute packing plans, we use a biased-randomized version of the Best-Fit heuristic (Burke et al. 2004). We integrate Monte Carlo (MC) simulation at two stages of the search process in order to assess the performance of the obtained solutions. More details are provided in Section 2. Our results prove that accounting for system's variability during the algorithm search yields not only to solutions with lower expected costs, but also to more robust or resilient solutions, i.e., solutions showing a lower variability than the ones obtained for the deterministic version of the problem (whenever they are applied in the stochastic environment). Additionally, our simheuristic approach could easily be adapted so, instead of searching for the solution with the minimum expected cost, it searches for the solution that maximizes the probability of not exceeding a specific cost threshold.

To the best of our knowledge, this is the first time in the literature that the 2L-VRP has been tackled considering the time-variability associated with real-life operations. The deterministic version of the 2L-VRP was originally introduced by Iori et al. (2007). The authors proposed an exact branch-and-cut algorithm to solve the routing aspects, and a branch-and-bound algorithm combined with heuristics and effective lower bounds to deal with the packing requirements. From the different loading configurations, the authors only tackle the sequential oriented case. In this variant, items cannot be rotated (oriented) and should be loaded in reverse order to the visited customers (sequential). Other variants of the 2L-VRP consider items rotation (non-oriented) and items rearrangement within the vehicle (unrestricted). The different combinations of these constraints provide four 2L-VRP variants. So far, only Fuellerer et al. (2009) have solved all four problem variants. In fact, only Fuellerer et al. (2009), Dominguez et al. (2014), and Zachariadis et al. (2016) have addressed the non-oriented loading configurations. The oriented versions of the problem have received far more attention in the literature (Gendreau et al. 2008; Leung et al. 2011; Duhamel et al. 2011; Zachariadis et al. 2013; Wei et al. 2015). Finally, some extensions of the 2L-VRP have also been proposed, considering oriented and non-oriented loading (Dominguez et al. 2014, Dominguez et al. 2015, Dominguez et al. 2016, Dominguez et al. 2016). In this work, we address the unrestricted oriented version 
of the problem, although the methodology proposed here can be extended to tackle other variants and extensions of the 2L-VRP.

\section{METHODOLOGY}

We propose a multi-start simheuristic that combines biased-randomized versions of classical routing and packing heuristics for solving the introduced stochastic variant of the 2L-VRP. Biased randomization is included at different stages in our method. As mentioned, the principle biased randomization builds upon consists of using skewed probability distributions to transform deterministic methods into probabilistic ones, while preserving the logic behind the original method (Faulin et al. 2008). In our approach, we apply biased-randomized techniques on the routing and packing heuristics. For the routing component, we use a modified version of the well-known Clarke and Wright (1964) heuristic. As for solving the packing, we use a biased-randomized version of the Best-Fit heuristic (Burke et al. 2004). In addition, we include MC simulation at two stages of our algorithm to deal with the stochastic nature of the problem. Figure 1 presents an overview of our algorithm.

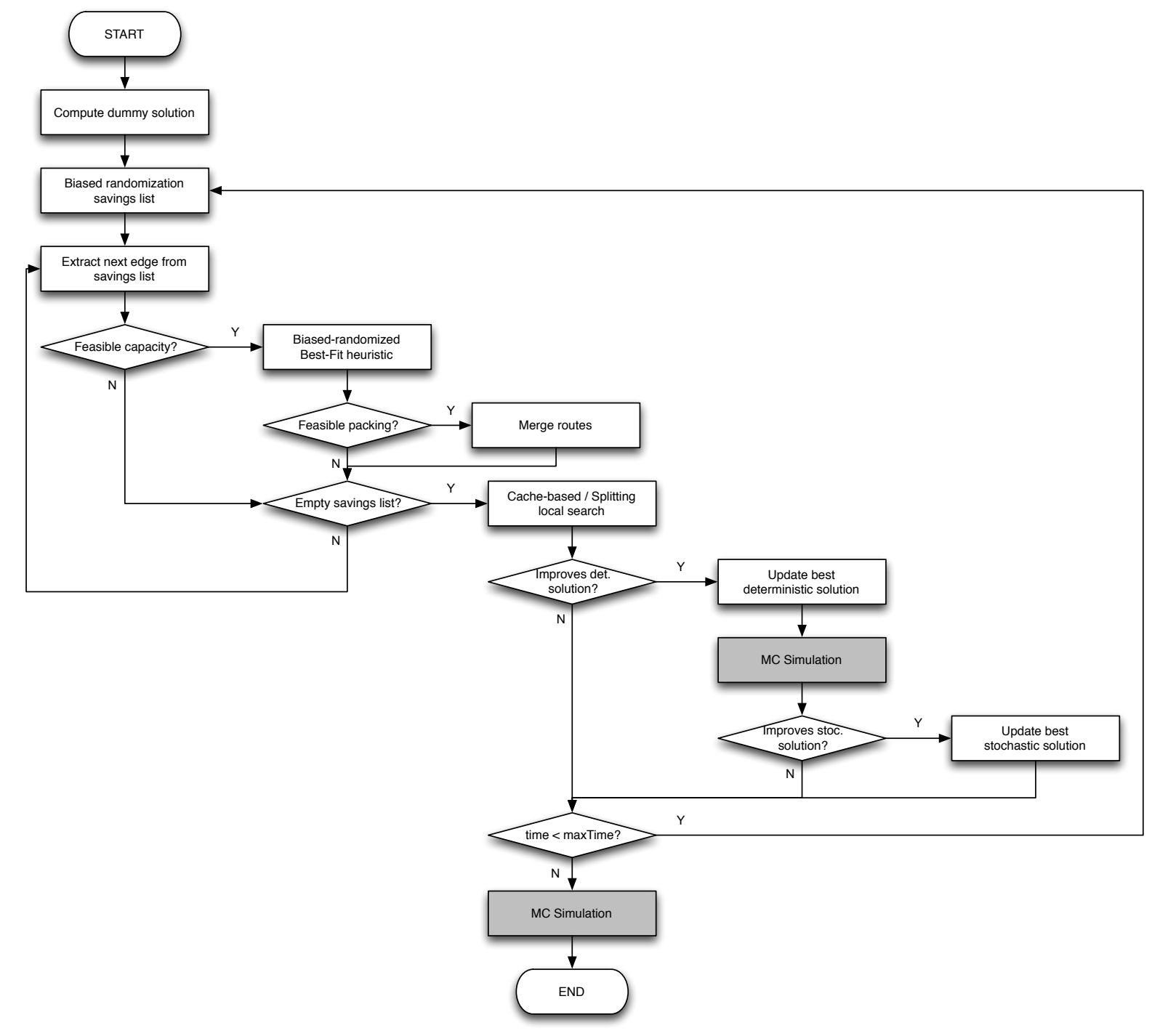

Figure 1: Diagram of the proposed multi-start simheuristic for the stochastic 2L-VRP. 
We start our method by generating an initial dummy solution as described in the savings heuristic by Clarke and Wright (1964). This initial solution consists of a return trip from the depot to each customer, using as many vehicles as the number of customers in the problem. Next, we compute the savings associated with each edge, i.e., the cost reduction of including an edge connecting two customers in one route instead of visiting them in two separate routes. These savings are then sorted in descending order. At this point, we initiate a multi-start process. At each iteration, we rearrange the elements in the savings list by applying a biased-randomized process, so edges associated with higher savings are more likely to be ranked at the top of the list. In our case, a geometric probability distribution, driven by a single parameter $\alpha(0<\alpha<1)$, is used to induce this skewed behavior. This process allows edges to be selected in a different order at each iteration of the multi-start process, while still preserving the behavior of the original heuristic. Then, the algorithm performs a route-merging process until the savings list is empty. At each iteration, the edge at the top of the list is selected. This edge connects two routes, which will be merged if: (i) there is enough weight capacity in the vehicle to carry all items from both routes; and (ii) they can be conveniently loaded without overlapping.

In order to evaluate packing feasibility, we use a multi-start biased-randomized version of the Best-Fit heuristic (Burke et al. 2004). The Best-Fit heuristic is a constructive and deterministic procedure that selects the next item (rectangle) to pack in the vehicle based on the bottom-left criterion, i.e.: among the available items, it always chooses the one that offers the 'best fit' when positioned at the bottom-left-most free position. We transform it into a biased-randomized by assigning probabilities to the different items, so that the better the fit the higher its probability of being chosen. Again, we use a geometric distribution to skew the selection, controlled by a single parameter $\beta(0<\beta<1)$. The multi-start process allows us to run the biased-randomized heuristic several times (maxPackIter parameter), thus increasing our chances of finding a feasible packing solution. We combine it with the use of a fast-access memory-based method (cache) to speed up the process. We check if the algorithm has already computed a feasible packing solution for the same configuration and, if so, routes are merged. Whenever the Best Fit heuristic finds a feasible solution, the packing cache memory is updated. In both cases, the packing process is stopped and the routing-construction resumes.

Note that, while other approaches propose a two-stage method -one for solving the packing problem and another for solving the routing component-, our approach integrates the packing problem as part of the routing-construction method. This way, we can guarantee the feasibility of all routes, as we explicitly take into account the loading constraints during the construction phase.

Eventually, we obtain a complete feasible solution. We then combine the use of memory-based and splitting techniques to further improve the solution (Juan et al. 2010). As in the packing case, we store previously computed routes and packing plans for a given set of customers in a fast-access cache memory. In this case, we keep in memory the best solution found so far for a specific set of customers to be visited. If the obtained solution contains the same set with a higher cost, the route stored in the cache memory is retrieved and the solution is updated. Otherwise, we add (or update) the route to the cache memory for subsequent iterations. The splitting technique constitutes a local search process similar to the large neighborhood search metaheuristic (Pisinger and Røpke 2010). This strategy first splits the solution into different disjoints (and feasible) parts according to geometric criteria, defining smaller 2L-VRP instances, and applies an iterative (maxSplitIter) routing-construction process similar to the one described. When all local solutions for each part are obtained, they are merged into an enhanced global solution. If this final solution improves the current best solution, the latter is updated with the new results.

As mentioned in Section 1, companies like Opein often have to deal with additional costs due to overtime and associated expenses. This problem is generally aggravated by unforeseen deviations from the original plan, e.g., due to longer traveling or servicing times. In order to account for these further expenses, we have included two additional terms in the cost function used to evaluate solutions. Given a set of nodes $I$ and a set of routes $R=\left\{r_{1}, r_{2}, \ldots, r_{m}\right\}$ visiting all customers, such as $r \subseteq I, \forall r \in R$, and $r_{1} \cap r_{2} \cap \ldots \cap r_{m}=\emptyset$, we define the cost of a route as $t_{r}=\sum_{i, j \in r} t_{i j}$, where $t_{i j}$ represents the traveling 
time from customer $i$ to customer $j$. Hence, the objective function of the classical 2L-VRP consists of minimizing the total traveling cost, i.e. $\sum_{r \in R} t_{r}$. In our case, we account for the excess traveling and service time above a certain threshold $\tau$ (e.g., 8 hours in application cases) and associated costs by defining the objective function as

$$
f(x)= \begin{cases}\sum_{r \in R} t_{r} & , \text { if } t_{r} \leq \tau, \forall r \in R \\ \sum_{r \in R} t_{r}+\rho \sum_{r \in R}\left(t_{r}-\tau\right)+\gamma & , \text { otherwise }\end{cases}
$$

where $\rho$ is the penalty for overtime and $\gamma$ is related to the associated fix costs.

So far, the computed solution responds to the deterministic 2L-VRP. In order to deal with the stochastic nature of the proposed problem, we include two simulation processes in our algorithm. In the first case, we run a short MC simulation accounting for traveling time variability whenever the best deterministic solution is improved. If results are able to improve the best stochastic solution found so far, the latter is updated. This way, we keep track of the most robust solution as the algorithm evolves. As the number of generated solutions can be large and the simulation process is time-consuming, we limit the number of iterations to be executed (maxShortIter). Once the algorithm is finished, we run a longer MC simulation (masLongIter) to better assess the best deterministic and stochastic solutions before reporting the final results. In both processes, we model the traveling time between any two customers $i$ and $j$ with a log-normal probability distribution, with a mean equivalent to the deterministic value $\left(\mu=t_{i j}\right)$ and a standard deviation $\sigma=k \mu$. As discussed in Juan et al. (2011), the log-normal distribution is quite convenient to model positive random variables such as customers' demands or traveling times.

Notice that an important advantage of our approach is its relative simplicity. Our method uses few parameters, significantly reducing its sensitivity to particular problem characteristics, providing a robust method able to perform efficiently across different instances. The values of the parameters were established after a quick tuning process in which different combinations of values were tested for a random sample of instances. After this process, the 'routing' biased-randomization parameter, $\alpha$, was set to 0.3; the 'packing' biased-randomization parameter, $\beta$, was set to a random number in the interval $(0.06,0.23)$; the maxPackIter and maxSplitIter parameters were set to be proportional to problems characteristics, i.e., number of customers and number of items; and, finally, simulation parameters maxShortIter and maxLongIter were set to 2,000 and 50,000, respectively.

\section{COMPUTATIONAL EXPERIMENTS}

We implemented the proposed algorithm using the Java programming language. All experiments were run in a standard PC with an Intel Core i5 processor at $3.4 \mathrm{GHz}$ and $4 \mathrm{~GB}$ RAM. The algorithm was executed on the Eclipse platform for Java over Windows 7.

The classical 2L-VRP benchmark set is composed by 180 instances proposed by Iori, Salazar, and Vigo (2007) and Gendreau et al. (2008), divided in 5 classes with 36 instances each. We extended these benchmark instances to the stochastic case in order to assess our methodology. As mentioned in Section 2, we selected a log-normal distribution to model traveling times between each pair of customers. With this extension, we can account for the significant variability in traveling and servicing times present in real systems. For our experiments, we defined the parameters of the log-normal distribution according to the values of each deterministic instance; i.e., the distribution mean $\mu$ is equal to the deterministic distance between customers $t_{i j}$, and the standard deviation $\sigma$ is proportional to such distance, $\sigma=k \mu=k t_{i j}$. We used $k=3$ in all the experiments included in this work.

Table 1 presents results for some instances of the extended stochastic $2 \mathrm{~L}-\mathrm{VRP}$ problem set. These figures correspond to the best results of 4 independent executions of our algorithm for each instance, using parameter values as described in Section 2. For each instance, we present the deterministic cost for the best deterministic and stochastic solutions, as well as the corresponding gap. Likewise, we include in the last three columns the stochastic value of the best deterministic and stochastic solutions, together with their gap. 
Table 1: Results for a subset of stochastic 2L-VRP instances.

\begin{tabular}{|lcc|rrr|rrr|}
\hline \multicolumn{3}{|c|}{ Instance } & \multicolumn{3}{c|}{ Deterministic 2L-VRP } & \multicolumn{3}{c|}{ Stochastic 2L-VRP } \\
\hline Name & Class & $\#$ & Det. Sol. & Stoc. Sol. & Gap (\%) & Det. Sol. & Stoc. Sol. & Gap (\%) \\
\hline E036-11h5 & 5 & 16 & 1295.01 & 1368.02 & 5.64 & 1696.42 & 1586.21 & -6.50 \\
E041-14h1 & 1 & 17 & 1342.95 & 1342.95 & 0.00 & 1884.46 & 1884.46 & 0.00 \\
E041-14h3 & 3 & 17 & 1338.36 & 1343.48 & 0.38 & 1858.05 & 1855.77 & -0.12 \\
E076-10e1 & 1 & 23 & 1890.54 & 2010.81 & 6.36 & 2490.33 & 2441.41 & -1.96 \\
E101-10c5 & 5 & 26 & 2722.98 & 2731.46 & 0.31 & 3347.91 & 3341.66 & -0.19 \\
E101-14s1 & 1 & 27 & 2291.24 & 2424.63 & 5.82 & 3163.21 & 3104.73 & -1.85 \\
\hline Average & & & & & 3.09 & & & -1.77 \\
\hline
\end{tabular}

We observe that integrating simulation in the search process is clearly able to improve solutions' robustness. As expected, considering the stochastic nature of some variables during search yields worse deterministic solutions (an average gap of 3.09\% with respect the deterministic approach). However, these same solutions show a better performance when variability is considered, with an average gap of $-1.77 \%$ with respect to the best deterministic solutions applied in stochastic instances. These results confirm that not accounting for variability during the search process may have a significant impact on the quality of the final solution. For instance, the direct application of deterministic solutions may provide lower costs in ideal situations, but will cause higher additional expenses in a real-life scenario. On the other hand, our approach is able to obtain more resilient solutions, which still may be able to perform reasonable well (3.09\% deviation) under ideal conditions. Moreover, this behavior is consistent across instances of different classes, as observed in Figure 2.

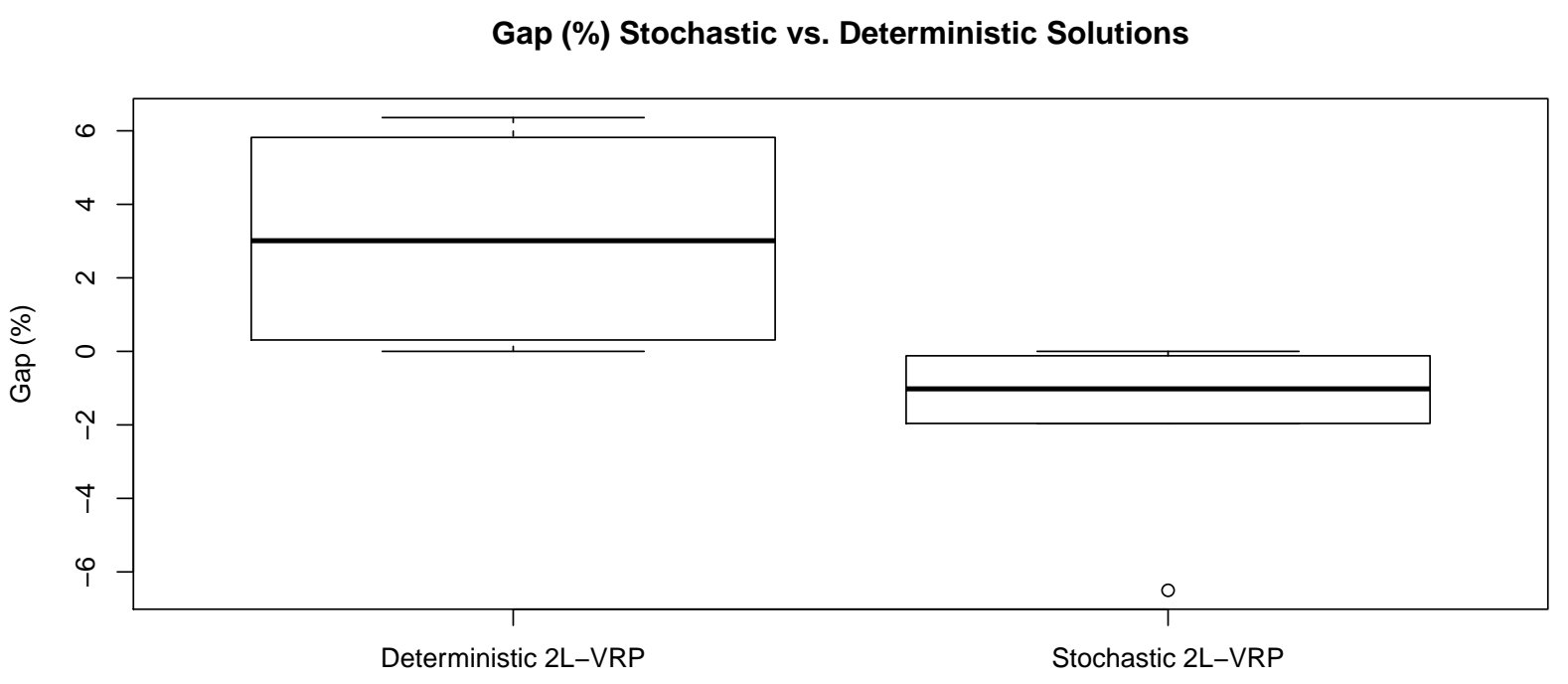

Figure 2: Gap (\%) distribution between the best stochastic and best deterministic solution, in equivalent deterministic and stochastic instances of the 2L-VRP.

\section{CONCLUSIONS}

The present paper presents a hybrid simheuristic for solving stochastic variants of the two-dimensional VRP (2L-VRP). This problem can be found in many real-life applications, like the one that motivated this 
work. In many cases, companies have to deal with uncertainty in some aspects of the problem, such as variable traveling and servicing times. Being the $2 \mathrm{~L}-\mathrm{VRP}$ a relatively new VRP variant in the literature, some of its extensions have not yet received attention from the research community. To the best of our knowledge, this is the first work addressing the stochastic version of the problem.

We propose a hybrid approach combining biased-randomized versions of classical routing and packing heuristics within a multi-start framework. Both heuristics are integrated in the routing-construction method, ensuring packing feasibility for all routes during the construction process, contrary to more traditional two-stage approaches. In order to deal with the stochastic nature of the problem, we embed Monte Carlo simulation at two stages of the search. This allows us to account for uncertainty in traveling and servicing times during search, aiming at providing more resilient solutions to scenarios with high variability. Our approach is enhanced by fast-access memory-based and splitting-based local search techniques, which help to improve solutions' quality and reduce computational times.

To test our methodology, we have generated stochastic instances of the 2L-VRP by modifying the ones contained in the classical benchmark sets, modeling traveling times as a log-normal probabilistic distribution. As we have proved, our methodology is able to find more resilient solutions, while still not far from the best found solution for ideal deterministic scenarios. Furthermore, our approach is able to perform consistently across adapted instances of the 2L-VRP.

Additional research is warranted in this stochastic version of the problem. Among the different research lines, the extension of our methodology to other 2L-VRP subvariants (e.g., sequential loading and/or items rotation) is to be studied in the near future. Indeed, the use of other metaheuristic frameworks and their integration with simulation techniques is especially promising. Finally, it is also of particular interest the study of further generalizations of the problem with direct applications in real-life, such as those including heterogeneous fleets or backhauling.

\section{ACKNOWLEDGMENTS}

This work has been partially supported by the Spanish Ministry of Economy and Competitiveness (TRA201348180-C3-P, TRA2015-71883-REDT), FEDER, and the Department of Universities, Research \& Information Society of the Catalan Government (2014-CTP-00001).

\section{REFERENCES}

Burke, E., G. Kendall, and G. Whitwell. 2004. "A new placement heuristic for the orthogonal stock-cutting problem". Operations Research 52:655-671.

Caceres-Cruz, J., P. Arias, D. Guimarans, D. Riera, and A. A. Juan. 2014. "Rich Vehicle Routing Problem: Survey". ACM Computing Surveys 47 (2): 1-28.

Clarke, G., and J. Wright. 1964. "Scheduling of vehicles from a central depot to a number of delivery points". Operations Research 12:568-581.

Dominguez, O., D. Guimarans, and A. A. Juan. 2015, November. "A hybrid heuristic for the 2L-VRP with clustered backhauls". In Proceedings of the XVI Conferencia de la Asociación Española para la Inteligencia Artificial (CAEPIA). Albacete, Spain.

Dominguez, O., D. Guimarans, A. A. Juan, and I. Nuez. 2016. "A Biased-Randomised Large Neighbourhood Search for the two-dimensional Vehicle Routing Problem with Backhauls". European Journal of Operational Research doi: 10.1016/j.ejor.2016.05.002.

Dominguez, O., A. A. Juan, B. Barrios, J. Faulin, and A. Agustin. 2014. "Using biased randomization for solving the two-dimensional loading vehicle routing problem with heterogeneous fleet". Annals of Operations Research doi: 10.1007/s10479-014-1551-4.

Dominguez, O., A. A. Juan, and J. Faulin. 2014. "A biased-randomized algorithm for the two-dimensional vehicle routing problem with and without item rotations". International Transactions in Operational Research 21:375-398. 
Dominguez, O., A. A. Juan, I. Nuez, and D. Ouelhadj. 2016. "An ILS-biased randomization algorithm for the two-dimensional loading HFVRP with sequential loading and items rotations". Journal of the Operational Research Society 67 (1): 37-53.

Duhamel, C., P. Lacomme, A. Quilliot, and H. Toussaint. 2011. "A multi-start evolutionary local search for the two-dimensional loading capacitated vehicle routing problem". Computers \& Operations Research 38 (3): 617-640.

Faulin, J., M. Gilibert, A. A. Juan, X. Vilajosana, and R. Ruiz. 2008. "SR-1: A simulation-based algorithm for the capacitated vehicle routing problem". In Proceedings of the 2008 Winter Simulation Conference, edited by S. J. Mason, R. R. Hill, L. Mönch, O. Rose, T. Jefferson, and J. W. Fowler, 2708-2716. Miami, FL, USA: Institute of Electrical and Electronics Engineers, Inc.

Fuellerer, G., K. Doerner, R. Hartl, and M. Iori. 2009. "Ant colony optimization for the two-dimensional loading vehicle routing problem". Computers \& Operations Research 36:655-673.

Gendreau, M., M. Iori, G. Laporte, and S. Martello. 2008. "A tabu search heuristic for the vehicle routing problem with two-dimensional loading constraints". Networks 51:4-18.

Golden, B., S. Raghavan, and E. Wasil. (Eds.) 2008. The Vehicle Routing Problem: Latest Advances and New Challenges. New York, NY: Springer.

Iori, M., J. Salazar, and D. Vigo. 2007. "An exact approach for the vehicle routing problem with twodimensional loading constraints". Transportation Science 41 (2): 253-264.

Juan, A. A., J. Faulin, A. Ferrer, H. Ramalhinho, and B. Barrios. 2013. "MIRHA: Multi-start biased randomization of heuristics with adaptive local search for solving non-smooth routing problems". TOP 21:109-132.

Juan, A. A., J. Faulin, S. Grasman, M. Rabe, and G. Figueira. 2015. "A review of Simheuristics: extending metaheuristics to deal with stochastic optimization problems". Operations Research Perspectives 2:6272.

Juan, A. A., J. Faulin, S. Grasman, D. Riera, J. Marull, and C. Mendez. 2011. "Using Safety Stocks and Simulation to Solve the Vehicle Routing Problem with Stochastic Demands". Transportation Research Part C (19): 751-765.

Juan, A. A., J. Faulin, R. Ruiz, B. Barrios, and S. Caballé. 2010. "The SR-GCWS hybrid algorithm for solving the capacitated vehicle routing problem". Applied Soft Computing 10 (1): 215-224.

Juan, A. A., H. Ramalhinho, M. Mateo, R. Luo, and Q. Castella. 2014. "Using iterated local search for solving the flow-shop problem: Parallelization, parametrization, and randomization issues". International Transactions in Operational Research 21:103-126.

Lahyani, R., M. Khemakhem, and F. Semet. 2015. "Rich vehicle routing problems: From a taxonomy to a definition". European Journal of Operational Research 241 (1): 1-14.

Leung, S., X. Zhou, D. Zhang, and J. Zheng. 2011. "Extended guided tabu search and a new packing algorithm for the two-dimensional loading vehicle routing problem". Computers \& Operations Research 38 (1): $205-215$.

Pisinger, D., and S. Røpke. 2010. "Large Neighborhood Search”. In Handbook of Metaheuristics, edited by M. Gendreau and J. Potvin, 399-419. New York, NY: Springer.

Toth, P., and D. Vigo. 2014. Vehicle Routing: Problems, Methods, and Applications. Philadelphia, PA: SIAM Publishers.

Wei, L., Z. Zhang, D. Zhang, and A. Lim. 2015. "A variable neighborhood search for the capacitated vehicle routing problem with two-dimensional loading constraints". European Journal of Operational Research 243 (3): 798-814.

Zachariadis, E., C. Tarantilis, and C. Kiranoudis. 2013. "Integrated distribution and loading planning via a compact metaheuristic algorithm". European Journal of Operational Research 228 (1): 56-71.

Zachariadis, E., C. Tarantilis, and C. Kiranoudis. 2016. "The vehicle routing problem with simultaneous pick-ups and deliveries and two-dimensional loading constraints". European Journal of Operational Research 251:369-386. 


\section{AUTHOR BIOGRAPHIES}

DANIEL GUIMARANS is an Associate Professor of the Aviation Academy at the Amsterdam University of Applied Sciences. He holds a PhD in Computer Science from the Autonomous University of Barcelona. His research interests are focused on hybridizing simulation with exact and heuristic optimisation techniques to solve stochastic problems and complex systems. Data analysis, and especially predictive and prescriptive analytics, also play a major role in his last research projects. Application areas of his research range from manufacturing to road and air transportation. His email address is d.guimarans.serrano@hva.nl.

OSCAR DOMINGUEZ is Operations Manager at Opein. He completed a $\mathrm{PhD}$ at the Universidad de Las Palmas de Gran Canaria, and has published several research articles on the two-dimensional vehicle routing problem. His e-mail address is oscar@opein.com.

ANGEL A. JUAN is Associate Professor at the Open University of Catalonia (Barcelona, Spain). He holds a PhD in Industrial Engineering and a MSc in Mathematics. He completed a pre-doctoral internship at Harvard University and a postdoctoral internship at the MIT Center for Transportation and Logistics. His research interests include applications of randomized algorithms and simheuristics in logistics, production, and Internet computing. He has published over 150 peer-reviewed papers in these fields. His website address is http://ajuanp.wordpress.com and his email address is ajuanp@uoc.edu.

ENOC MARTINEZ is an Assistant Researcher of the ICSO group at the Internet Interdisciplinary Institute (IN3). He is a highly skilled programmer who is currently completing his MSc in Computer Science. As a programmer, he has won several contests on algorithm programming in Spain. His e-mail address is e@enocmartinez.com. 